\title{
Gamma-rays from accretion process onto millisecond pulsars
}

\author{
Wlodek Bednarek* \\ Department of Astrophysics, University of Łódź \\ E-mail: bednareuni.lodz.pl
}

\begin{abstract}
The transition states between the rotation powered and accretion powered non-thermal emission in millisecond pulsar binary systems have been recently observed in the case of three objects PSR J1023+0038, PSR J1824-2452, and PSR J1227-4859. The transition is related to the significant change in the $\gamma$-ray flux. The origin of this enhanced emission seems to be related to the penetration of the inner pulsar magnetosphere by the accretion disk. We propose that the radiation processes, characteristic for the rotation powered pulsar, can co-exist with the presence of an accretion disk in the inner pulsar magnetosphere. The $\gamma$-ray emission can be also produced by secondary leptons in the Compton scattering of thermal radiation from the accretion disk. The accretion disk is assumed to penetrate deep into the pulsar magnetosphere allowing the matter to fall onto the NS surface producing pulsed X-ray emission. We show that the sum of the rotation powered pulsar $\gamma$-ray emission, produced by the primary electrons in the curvature process, and the $\gamma$-ray emission, produced by secondary leptons, can explain the observed high energy radiation from the redback binary pulsar PSR J1227-4853 in the high state.
\end{abstract}

The 34th International Cosmic Ray Conference,

30 July- 6 August, 2015

The Hague, The Netherlands

\footnotetext{
* Speaker.
} 


\section{Introduction}

The hypothesis on the formation of millisecond pulsars (MSPs) in binary systems has recently got strong support with the discovery of three MSP binary systems that showed transition from the rotation powered pulsar state to the accretion disk powered state. The binary system containing PSR J1023+0038 has shown at first the evidences of an accretion disk with a relatively low power $\left(<10^{35} \mathrm{erg} \mathrm{s}^{-1}\right.$ ) which moved to the rotation powered pulsar with clear radio pulsations (Archibald et al. 2009). Similar behaviour has been also observed in the binary containing PSR J1824-2452 (Papitto et al. 2013). X-ray pulsations with the period of the pulsar have been observed in PSR J1824-2452 (Papitto et al. 2013). This last result indicates that the matter can reach the NS surface also at low accretion rates. The mentioned binary systems belong to the redback class which contain companion stars with the mass $\sim 0.2 \mathrm{M}_{\odot}$ accreting matter through the 1st Lagrangian point. Thus, the formation of the accretion disks around these pulsars is expected. The transition between two states in the binary systems containing PSR J1023+0038 and PSR J1227-4853 is connected with the change of the $\gamma$-ray flux which is a factor of a few larger in the state with the presence of the accretion disk (Takata et al. 2014, Xing \& Wang 2014, Johnson et al. 2015).

The high luminosity different $\gamma$-ray states has been explained in terms of two models. Takata et al. (2014) assumes that the accretion disk does not penetrate below the light cylinder radius of the pulsar magnetosphere due to its evaporation by $\gamma$-ray radiation produced in the outer gap (Takata et al. 2010) or as a result of the disk interaction with the pulsar wind (Burderi et al. 2001). Papitto et al. (2014) proposes that the pulsar mechanism is quenched by the accretion disk which penetrates below the light cylinder radius and $\gamma$-rays in the disk state are produced in the synchrotron selfCompton process by electrons accelerated on the border between the rotating magnetosphere and the inner accretion disk (see also Bednarek 2009). Here we consider another scenario in which the accretion disk slowly penetrates the inner pulsar magnetosphere up to the co-rotation radius, allowing the matter to fall onto the NS surface. However, the acceleration gaps in the pulsar magnetosphere, which developed at large distance from the disk (e.g. as expected in terms of the slot gap model), are not switched off. Due to the geometry of the magnetic field outside the light cylinder radius, the matter from the disk cannot penetrate into gaps at large distance from the disk surface. We propose that secondary leptons, produced in the region of the slot gap, comptonize disk radiation producing enhanced $\gamma$-ray emission in the $\mathrm{GeV}$ energies (for details see Bednarek 2015).

\section{A model for the high energy radiation in the disk state}

We concentrate on the redback binary system containing millisecond pulsar PSR J1227-4853. The post-transition $\gamma$-ray emission from this binary system shows clear modulation with the period of the pulsar (Johnson et al. 2015). Therefore, it is expected to be produced in terms of rotation powered pulsar mechanism. According to the outer gap and slot gap models this emission mostly comes from the region close to the light cylinder radius and originates in the curvature process of primary leptons accelerated in the gap to the Lorentz factors of the order of a few $10^{6}$. On the other hand, the $\gamma$-ray emission in the pre-transition state, in which the accretion disk is present, is proposed to be produced in another mechanism. Here we consider the scenario for the pre-transition emission state in the binary PSR J1227-4853. We assume that the accretion disk can penetrate 
below the light cylinder radius but it does not switch off completely the pulsar mechanism. This can happen since in the case of nearly aligned rotator the plasma in the accretion disk is not directly connected by the magnetic field lines to the regions in the inner pulsar magnetosphere which are at large distances from the equatorial plane. It is expected that the density of matter outside the accretion disk in the wind or an extended corona is much rare than the density of matter within the disk. Therefore, its energy density is likely to be also lower than the energy density of the magnetic field in the pulsar wind zone. The slot gap appears in the pulsar magnetosphere as a result of the space charge limited outflow scenario (Arons \& Scharleman 1979, Harding \& Muslimov 1998). In this model the matter from the NS surface is not expected to saturate the electric field of the gap since plasma with Goldreich \& Julian (1969, GJ) density at the NS surface drops below GJ density at some distance from the surface due to the dipole geometry of the magnetic field lines. Thus, the conditions for the appearance of the accelerating gap are satisfied. Along the magnetic field lines above the null surface, electrons can be accelerated in the slot gap scenario. As expected in classical pulsar model, we assume that the post-transition $\gamma$-ray emission is due to curvature radiation of the primary electrons accelerated in the electric field of the slot gap model (e.g. Arons 1983). A part of these primary curvature $\gamma$-rays is absorbed close to the gap and creates the secondary population of $e^{ \pm}$plasma. Additional disk radiation field can significantly increase the population of secondary leptons due to absorption of primary gamma-rays in the X-ray radiation as postulated by e.g. Romani (1996). These secondary leptons comptonize thermal radiation from the nearby accretion disk penetrating deep into the inner pulsar magnetosphere. In such scenario, the pretransition $\gamma$-ray emission state is the sum of the $\gamma$-ray emission produced in the inverse Compton process of secondary leptons and the lower level $\gamma$-ray emission produced in the pulsar mechanism (observed also in the post-transition state). We suppose that the production of $\gamma$-rays in terms of the the outer gap model (Cheng et al. 1986) may be impossible in such scenario. Note that the accretion disk, penetrating the inner pulsar magnetosphere, is quite close to the outer gap at the light cylinder radius. Thus, a part of the matter from the disk could easily penetrate into the outer gap switching off its electric field.

The transition states are expected to be initialized by the enhanced accretion rate from the stellar companion of the pulsar due to the Roche lobe overflow. The rotational axis of the pulsar should be close to alignment to the rotational axis of the binary system due to the past accretion history of the accreting matter. Therefore, the matter is expected to penetrate the outer pulsar magnetosphere along the rotational plane of the binary system. In the transition state the pressure of the matter, accreting in the rotational plane, is not balanced by the pressure of the pulsar wind. Therefore, accretion disk starts to build up. With accumulation of matter in the accretion disk, its inner radius shrinks reaching at first the light cylinder radius and after that it can penetrate into the inner pulsar magnetosphere. The location of the disk inner radius in the pulsar magnetosphere (below the light cylinder) can be estimated by balancing the kinetic energy density of the plasma in the disk with the magnetic field energy density, $B^{2}(R) / 8 \pi=\rho v_{\mathrm{K}}^{2} / 2$, where $B(R)=B_{\mathrm{NS}}\left(R_{\mathrm{NS}} / R\right)^{3}$ is the magnetic field strength at the distance $R$ from the centre of the NS, $v_{\mathrm{K}}=\left(G M_{\mathrm{NS}} / R\right)^{1 / 2}$ is the Keplerian velocity of the matter in the accretion disk, $R_{\mathrm{NS}}$ and $B_{\mathrm{NS}}=10^{8} B_{8} \mathrm{G}$ are the radius of NS and its surface magnetic field strength, $\rho$ is the density of matter at the inner disk radius $R_{\text {in }}$ (in grams). The above condition allows us to estimate the location of the so called Alfven radius, $R_{\mathrm{A}}=8.4 \times 10^{4}\left(B_{8}^{2} / \rho\right)^{1 / 5} \mathrm{~cm}$, where $\rho$ is in grams. In fact, the inner disk radius is expected 
to be located at the magneto-spheric radius, $R_{\mathrm{m}}$, which is not precisely known fraction of the Alfven radius, $R_{\mathrm{m}}=\chi R_{\mathrm{A}} \cdot \chi$ is expected to lay in the range $\chi \sim 0.1-1$ (see e.g. Lamb, Pethick $\&$ Pines 1973, and more recent discussion of this value in Bozzo et al. (2009). Even for relatively low density of accreting matter, the disk can penetrate the inner pulsar magnetosphere below the light cylinder radius. Note that the Alfven radius slowly shrinks with the increasing density of the matter in the inner disk. In this stage the accretion disk resembles the models of the dead disks (Sunyaev \& Shakura 1977) or trapped disks (Spruit \& Taam 1993, D’Angelo \& Spruit 2010, 2012) around compact objects in which substantial amount of matter can be accumulated but the thermal structure of the disk is determined by the continuously supplied fresh matter through the 1st Lagrangian point of the binary system. The process of building up of the disk continue up to the moment when the magneto-spheric radius reaches so called co-rotation radius defined as the distance from NS at which the Keplerian velocity of matter becomes comparable with the rotational velocity of the magnetic field lines, $v_{\mathrm{K}}=v_{\mathrm{rot}}$, where $v_{\mathrm{rot}}=2 \pi R / P_{\mathrm{NS}}, P_{\mathrm{NS}}=10^{-3} P_{\mathrm{ms}} \mathrm{s}$ and $M_{\mathrm{NS}}$ are the pulsar rotational period and its mass assumed to be $1.4 \mathrm{M}_{\odot}$. From this moment, the matter from the disk can fall onto the surface of NS. The co-rotation radius is located at, $R_{\text {cor }}=$ $\left(G M_{\mathrm{NS}}\right)^{1 / 3}\left(P_{\mathrm{NS}} / 2 \pi\right)^{2 / 3} \approx 1.7 \times 10^{6} P_{\mathrm{ms}}^{2 / 3} \mathrm{~cm}$. For $R_{\mathrm{m}} \approx R_{\mathrm{cor}}$, the matter starts to fall onto the NS following local magnetic field lines. This distance defines also in our scenario the inner radius of the accretion disk. If the magneto-spheric radius is always lower than the co-rotation radius then the disk extends up to the surface of NS.

In the quasi-steady state, the matter flows through the accretion disk at the rate defined by the accretion rate of the matter passing through the 1st Lagrangian point. We assume that this accretion energy is irradiated from the disk surface as expected in the Shakura \& Sunyaev (1973) model. Then, the disk luminosity is $L_{\mathrm{D}}=G M_{\mathrm{NS}} \dot{M} / 2 R_{\mathrm{in}}=4 \pi R_{\mathrm{in}}^{2} \sigma_{\mathrm{SB}} T_{\mathrm{in}}^{4}=10^{34} L_{34} \mathrm{erg} \mathrm{s}^{1}$, where $\dot{M}$ is the accretion rate. Based on this model, we estimate the characteristic temperature of the disk at the inner radius on, $T_{\text {in }} \approx 1.9 \times 10^{6} L_{34}^{1 / 4} \mathrm{~K}$, if the disk extends up to the NS surface (then $\left.R_{\mathrm{m}}<R_{\text {cor }}\right)$ and on, $T_{\mathrm{in}} \approx 1.5 \times 10^{6} L_{34}^{1 / 4} / P_{\mathrm{ms}}^{1 / 3} \mathrm{~K}$, if the disk extends only to the co-rotation radius (then $R_{\mathrm{m}} \approx R_{\text {cor }}$ ).

The radiation field in the slot gap has been calculated assuming the Sunyaev \& Shakura disk model in which the gravitational energy extracted by the plasma is locally irradiated from the disk surface. In this model the temperature profile on the disk surface is given by approximate formula $T(R) \approx T_{\text {in }}\left(R_{\text {in }} / R\right)^{3 / 4}$. We calculate the density of photons in the slot gap by integrating over the disk temperature profile following prescription given in e.g. Frank et al. (1985, see Eq. 5.43). The maximum power is radiated from the surface with temperature $T_{\text {in }}$ at photon energies $\varepsilon \sim$ $3 k_{\mathrm{B}} T_{\text {in }} \approx 390\left(L_{34} / P_{\mathrm{ms}}^{3}\right)^{1 / 4} \mathrm{eV}$. For the disk luminosity $3 \times 10^{33} \mathrm{erg} \mathrm{s}^{-1}$ and the pulsar period 1.7 $\mathrm{ms}, \varepsilon$ is $\sim 200 \mathrm{eV}$, falling into the UV energy range. Such photons are comptonized on the border between the Thomson $(\mathrm{T})$ and the Klein-Nishina $(\mathrm{KN})$ regimes by electrons with energies $E_{\mathrm{T} / \mathrm{KN}}=$ $m_{\mathrm{e}}^{2} / \varepsilon \sim 0.7\left(P_{\mathrm{ms}}^{3} / L_{34}\right)^{1 / 4} \mathrm{GeV}$. Therefore, in the calculations of the IC spectra, the $\mathrm{KN}$ effects has to be taken into account. The mean free path for electrons (in the Thomson regime) on scattering the thermal radiation from the disk with characteristic temperature $T_{\mathrm{in}}$ is, $\lambda_{\mathrm{IC} / \mathrm{T}}=\left(\sigma_{\mathrm{T}} n_{\mathrm{ph}}\right)^{-1} \approx$ $2.7 \times 10^{5} P_{\mathrm{ms}}^{5 / 3} / L_{34}^{3 / 4} \mathrm{~cm}$, where $n_{\mathrm{ph}} \approx 380\left(T_{\mathrm{in}} / 2.7 \mathrm{~K}\right)^{3}\left(R_{\mathrm{in}} / R_{\mathrm{LC}}\right)^{2} \mathrm{ph} \mathrm{cm}^{-3}$ is the density of thermal photons from the disk in the location of the slot gap close to the light cylinder radius, and $\sigma_{\mathrm{T}}$ is the Thomson cross section. This distance scale is smaller than the distance scale for the propagation 


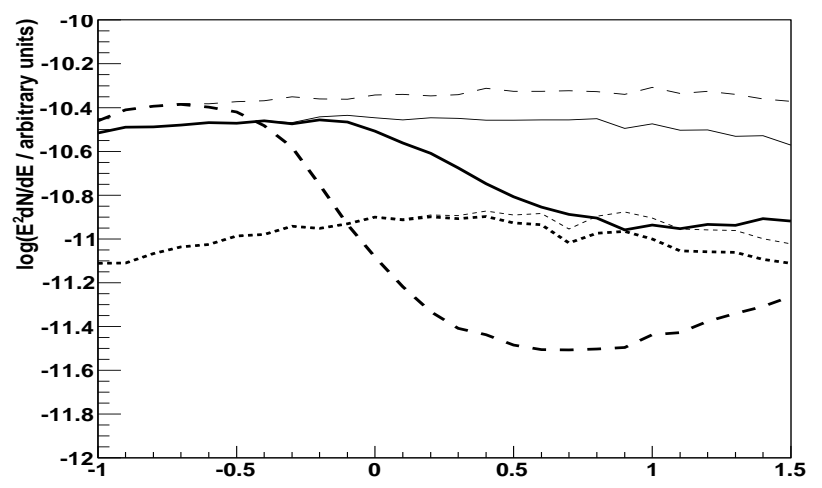

Figure 1: Spectral Energy Distribution (SED, differential spectra multiplied by energy squared) of $\gamma$-rays produced in the IC scattering of the accretion disk radiation by secondary leptons with the power law spectrum and spectral index equal to -2 between $100 \mathrm{MeV}$ and $100 \mathrm{GeV}$ (thin curves) and the spectra after absorption of $\gamma$-rays in the disk radiation (thick curves). Specific spectra are calculated for luminosities of the accretion disk $L_{\mathrm{D}}=10^{33} \mathrm{erg} \mathrm{s}^{-1}$ (dotted), $10^{34} \mathrm{erg} \mathrm{s}^{-1}$ (solid), and $10^{35} \mathrm{erg} \mathrm{s}^{-1}$ (dashed). The disk extends up to the co-rotation radius and the period of the pulsar is equal to $1.7 \mathrm{~ms}$ (Bednarek 2015).

of secondary leptons in the inner pulsar magnetosphere given by the light cylinder radius for the disk luminosity above, $L_{\min } \approx 2.2 \times 10^{32} P_{\mathrm{ms}}^{8 / 9} \mathrm{erg} \mathrm{s}^{-1}$. We also calculate the optical depths for absorption of curvature $\gamma$-rays, produced by primary electrons, and the IC $\gamma$-ray spectra, produced by secondary leptons, in the radiation field of the accretion disk. The absorption of $\gamma$-rays strongly depend on the accretion rate (and so the disk luminosity). Since the $\gamma$-ray production region in the slot gap is assumed to be homogeneous, with the characteristic dimension equal to $R_{\mathrm{LC}}$, the absorption effects are approximately included by multiplying produced $\gamma$-ray spectra by the factor equal to $(1-\exp (-\tau)) / \tau$. We show the example calculations of the $\gamma$-ray spectra produced in terms of such model including the effects of their absorption (Fig. 1). It is clear that absorption of $\gamma$-rays starts to be important for the disk luminosities above $\sim 10^{33} \mathrm{erg} \mathrm{s}^{-1}$.

\section{Gamma-ray emission states in binary system PSR J1227-4853}

We assumed that enhanced $\gamma$-ray emission in the pre-transition state in PSR J1227-4853 is produced by the secondary $e^{ \pm}$leptons which up-scatter disk thermal radiation to $\mathrm{GeV}$ energies. In fact, $e^{ \pm}$pairs should be present in the region of the slot gap of the pulsar since in the posttransition state a clear radio pulsations are observed. The $\gamma$-ray spectrum in the post-transition state is expected to originate in the curvature process of primary electrons accelerated in the electric field of the slot gap. A large amount of secondary leptons is expected to be produced with close to the power law spectrum in the energy range between $100 \mathrm{MeV}$ and $100 \mathrm{GeV}$. In fact, such type of secondary leptons spectra are expected to be produced in the pulsar magneto-spheres in the case of the existence of the extended polar cap regions (see e.g. Hibschman \& Arons 2001). In the pretransition state, the accretion disk penetrates deep into the inner pulsar magnetosphere providing additional soft photon target to be up-scattered by secondary leptons. We simulate the propagation of leptons on the distance scale of the light cylinder radius in the thermal radiation field from the inner accretion disk. The synchrotron energy losses of leptons are taken into account assuming 


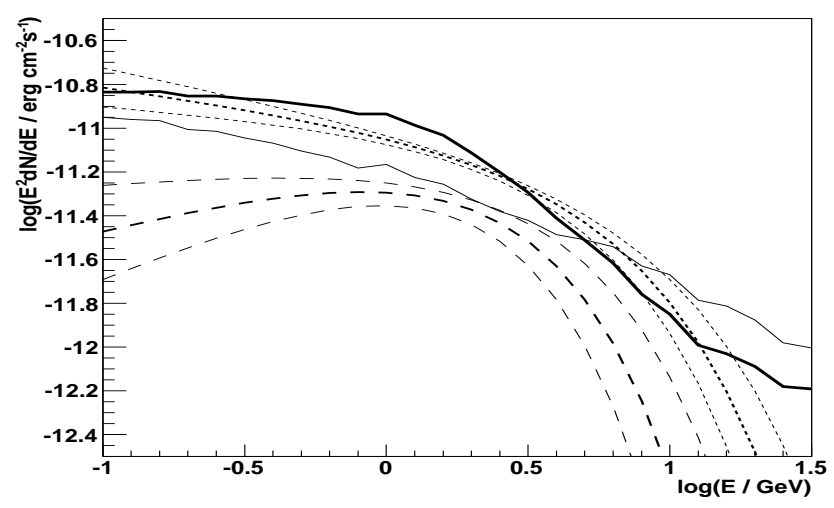

Figure 2: Spectral Energy Distribution (SED) of the Redback type binary system containing MSP PSR J1227-4853. The approximation of the pre-transition and post-transition spectra from PSR J1227-4853 (dotted and dashed curves, see Johnson et al. 2015). The IC spectrum produced by secondary $e^{ \pm}$pairs which comptonize thermal radiation from the accretion disk, before (thin solid curve) and after absorption in the disk radiation (thick solid). It is assumed that the secondary $\mathrm{e}^{ \pm}$spectrum is of a simple power law type with the spectral index -2.6 between $0.1-100 \mathrm{GeV}$. The accretion disk luminosity is assumed to be equal to $L_{\mathrm{D}}=3 \times 10^{33} \mathrm{erg} \mathrm{s}^{-1}$ (Bednarek 2015).

their average pitch angle of of the order of $0.1 \mathrm{rad}$. For the magnetic field strength of the NS at the light cylinder radius, the synchrotron energy losses of leptons are estimated to be clearly lower than their energy losses on the IC process in the accretion disk radiation field.

The $\gamma$-ray spectrum in the pre-transition state is reasonably well described by the sum of the spectrum from the post-transition state (curvature radiation of primary electrons) and the spectrum produced by secondary leptons in the inverse Compton scattering of disk radiation. This fitting to the pre-transition $\gamma$-ray spectrum has been obtained for the simple assumption on the spectrum of secondary leptons which has been assumed to be of the power law type with the spectral index equal to -2.6 between $100 \mathrm{MeV}$ and $100 \mathrm{GeV}$ and the disk radiation field which luminosity is equal to $3 \times 10^{33} \mathrm{erg} \mathrm{s}^{-1}$ (see Fig. 3). In order to obtain correct $\gamma$-ray luminosity in the pre-transition state, the power in secondary leptons should be equal to $\sim 0.33$ of the product of the the pulsar spin down luminosity (equal to $9 \times 10^{34} \mathrm{erg} \mathrm{s}^{-1}$ in the case of PSR J1227-4853) and the collimation factor of the $\gamma$-ray emission produced by leptons in the slot gap.

Since the secondary leptons have some degree of beaming it is possible that the $\gamma$-ray emission during pre-transition state can also show some modulation with the period of the pulsar. However the presence of the accretion disk can also influence the pulsar characteristic spin down rate which might complicate direct detection of such modulation.

\section{Conclusion}

Observations suggest that the disk has to penetrate deep into the inner pulsar magnetosphere and that at least some of the disk matter has to fall onto the neutron star surface. We propose that high $\gamma$-ray emission state from MSP binary systems, observed in the presence of the accretion disk, is produced in the comptonization process of the disk radiation by secondary leptons created relatively far from the disk in the region of the slot gap. In contrast to the outer gap scenario, $e^{ \pm}$ 
pairs in the slot gap, are expected to be produced copiously since that acceleration region is far away from the disk. In this scenario, $\gamma$-rays in the rotation powered state originate in the curvature process of primary electrons. In the disk state, primary electrons produce curvature $\gamma$-rays, which can be additionally absorbed in the disk radiation ejecting secondary leptons in the cascade process occurring above the null surface. These secondary leptons are expected to have a few orders of magnitude lower energies than primary electrons. Therefore, they are able to scatter efficiently UV to soft X-ray radiation produced by the accretion disk in contrast to primary electrons. We simulated propagation and radiation process of secondary leptons, in the region close to the light cylinder radius and above the null surface. The accretion disks with luminosities in the range expected for the accreting millisecond pulsars are considered. We take into account IC scattering of disk radiation, synchrotron losses of secondary leptons and also absorption of $\gamma$-rays in the disk radiation. The results of calculations of the IC $\gamma$-ray spectra are compared with the two $\gamma$-ray emission states from the transiting MSP Redback type binary system J1227-4853 for which the spectral information in the $\gamma$-ray energy range has been precisely measured (Johnson et al. 2015). Reasonable description has been obtained in terms of such model for likely parameters of this system.

Another transitional millisecond pulsar, PSR J1023+0038, shows the flux change in the $\gamma$-rays by a factor of $\sim 5$ in respect to the change by a factor of $\sim 2$ in the case of discussed here source. We think that such large flux changes between different states could be also understood in terms of the proposed scenario. In principle the power in secondary leptons, responsible for additional component in the disk state, can be larger than the power in observed $\gamma$-rays. The primary $\gamma$-rays can be efficiently absorbed in the gap region and only a small part can survive this process and escape to the observer. These leptons meet strong radiation field from the accretion disk allowing them efficient conversion of their energy to the IC $\gamma$-ray component observed in the disk state of transitional pulsars. Moreover the emission geometry can also play an important role. The rotating disk plasma is expected to produce additional component of the magnetic field which might slightly influence the dipole component of NS. As a result, the structure of magnetosphere at the light cylinder can change allowing enhanced $\gamma$-ray emission at certain directions.

\section{Acknowledgements}

This work is supported by the grant through the Polish Narodowe Centrum Nauki No. 2011/01/B/ST9/00411.

\section{References}

[1] Archibald, A.M. et al. A Radio Pulsar/X-ray Binary Link Science 324 (2009) 1411

[2] Arons, J. Pair creation above pulsar polar caps - Geometrical structure and energetics of slot gaps ApJ 266 (1983) 215

[3] Arons, J., Scharleman, E.T. Pair formation above pulsar polar caps - Structure of the low altitude acceleration zone ApJ 231 (1979) 854

[4] Bednarek, W. Gamma-rays from the vicinity of accreting neutron stars inside compact high-mass X-ray binaries A\&A 495 (2009) 919 
[5] Bednarek, W. 2015 Gamma-ray emission states in the redback millisecond pulsar binary system PSR J1227-4853 MNRAS in press (2015) [arXiv:1505.01940]

[6] Bozzo, E. et al. Can disk-magnetosphere interaction models and beat frequency models for quasi-periodic oscillation in accreting X-ray pulsars be reconciled? A\&A 493 (2009) 809

[7] Burderi, L. et al. Where May Ultrafast Rotating Neutron Stars Be Hidden? ApJL 560 (2001) L71

[8] Cheng, K.S. et al. Energetic radiation from rapidly spinning pulsars. I - Outer magnetosphere gaps. II - VELA and Crab ApJ 300 (1986) 500

[9] D’Angelo, C.R., Spruit, H.C. Episodic accretion on to strongly magnetic stars MNRAS 406 (2010) 1208

[10] D'Angelo, C.R., Spruit, H.C. Accretion discs trapped near corotation MNRAS 420 (2012) 416

[11] Frank, J.F. et al. in Accretion Power in Astrophysics Cambridge University Press (1985)

[12] Goldreich, P., Julian, W.H. Pulsar Electrodynamics ApJ 157 (1969) 869

[13] Harding, A.K., Muslimov, A.G. Particle Acceleration Zones above Pulsar Polar Caps: Electron and Positron Pair Formation Fronts ApJ 508 (1998) 328

[14] Hibschman, J.A., Arons, J. Pair Production Multiplicities in Rotation-powered Pulsars ApJ 560 (2001) 871

[15] Johnson, T.J. et al. Discovery of Gamma-Ray Pulsations from the Transitional Redback PSR J1227âĂŞ4853 ApJ 806 (2015) 91

[16] Lamb, F.K., Pethick, C.J., Pines, D. A Model for Compact X-Ray Sources: Accretion by Rotating Magnetic Stars ApJ 184 (1973) 271

[17] Papitto, A. et al. Swings between rotation and accretion power in a binary millisecond pulsar Nature 501 (2013) 517

[18] Papitto, A., Torres, D.F., Li, J. A propeller scenario for the gamma-ray emission of low-mass X-ray binaries: the case of XSS J12270-4859 MNRAS 438 (2014) 2105

[19] Romani, R.W. Gamma-Ray Pulsars: Radiation Processes in the Outer Magnetosphere ApJ 470 (1996) 469

[20] Spruit, H.C., Taam, R.E. An instability associated with a magnetosphere-disk interaction ApJ 402 (1993) 593

[21] Shakura, N.I., Sunyaev, R.A. Black holes in binary systems. Observational appearance A\&A 24 (1973) 337

[22] Sunyaev, R.A., Shakura, N.I. Disk reservoirs in binary systems and prospects for observing them PAZh 3 (1977) 262

[23] Takata, J. et al. On the Transition from Accretion-powered to Rotation-powered Millisecond Pulsars ApJL 723 (2010) L68

[24] Takata, J. et al. Multi-wavelength Emissions from the Millisecond Pulsar Binary PSR J1023+0038 during an Accretion Active State ApJ 785 (2014) 131

[25] Xing, Y., Wang, Z. Fermi Observation of the transitional pulsar binary XSS J12270-4859 ApJ (2014) [arXiv:1411.3449] 